\title{
The awareness of novelty for strangely familiar words: A laboratory analogue of the déjà vu experience
}

Déjà vu is a nebulous memory experience defined by a clash between evaluations of familiarity and novelty for the same stimulus. We sought to generate it in the laboratory by pairing a DRM recognition task, which generates erroneous familiarity for critical words, with a monitoring task by which participants realise that some of these erroneously familiar words are in fact novel. We tested 30 participants in an experiment in which we varied both participant awareness of stimulus novelty and erroneous familiarity strength. We found that déjà vu reports were most frequent for high novelty critical words ( $25 \%)$, with low novelty critical words yielding only baseline levels of déjà vu report frequency ( 10\%). There was no significant variation in déjà vu report frequency according to familiarity strength. Discursive accounts of the experimentally-generated déjà vu experience suggest that aspects of the naturalistic déjà vu experience were captured by this analogue, but that the analogue was also limited in its focus and prone to influence by demand characteristics. We discuss theoretical and methodological considerations relevant to further development of this procedure and propose that verifiable novelty is an important component of both naturalistic and experimental analogues of déjà vu. 
The awareness of novelty for strangely familiar words:

A laboratory analogue of the déjà vu experience.

5 Correspondence concerning this article should be addressed to Akira O'Connor, School of

6 Psychology, University of St Andrews, St Mary's College, South Street, St Andrews, Fife, KY16

7 9JP, Scotland, UK. E-mail: aro2@st-andrews.ac.uk 


\section{Abstract}

9 Déjà vu is a nebulous memory experience defined by a clash between evaluations of familiarity

10 and novelty for the same stimulus. We sought to generate it in the laboratory by pairing a DRM

11 recognition task, which generates erroneous familiarity for critical words, with a monitoring task

12 by which participants realise that some of these erroneously familiar words are in fact novel. We

13 tested 30 participants in an experiment in which we varied both participant awareness of

14 stimulus novelty and erroneous familiarity strength. We found that déjà vu reports were most

15 frequent for high novelty critical words ( 25\%), with low novelty critical words yielding only

16 baseline levels of déjà vu report frequency $(\sim 10 \%)$. There was no significant variation in déjà vu

17 report frequency according to familiarity strength. Discursive accounts of the experimentally-

18 generated déjà vu experience suggest that aspects of the naturalistic déjà vu experience were

19 captured by this analogue, but that the analogue was also limited in its focus and prone to

20 influence by demand characteristics. We discuss theoretical and methodological considerations

21 relevant to further development of this procedure and propose that verifiable novelty is an

22 important component of both naturalistic and experimental analogues of déjà vu. 


\section{A laboratory analogue of the déjà vu experience.}

25 Déjà vu is defined as a "clash between two simultaneous and opposing mental evaluations: an

26 objective assessment of unfamiliarity with a subjective evaluation of familiarity" (p.2 Brown,

27 2004). The sensation has captured public interest, e.g. its discussion in Heller's (1961) 'Catch

2822 ' and use as a plot device in 'The Matrix' (Silver, Wachowski \& Wachowski, 1999), but its

29 scientific investigation remains sparse, perhaps because the sensation is fleeting and occurs

30 unpredictably. Despite its volatility, the experience is by no means uncommon - surveys usually

31 find lifetime incidences in excess of $65 \%$, with young adults likely to report multiple yearly

32 experiences (Brown, 2003). As such, déjà vu presents a window into the healthy memory

33 decision-making process through study of perturbations to the signals it must adjudicate

34 between.

35 Much of the research that has examined déjà vu has used the clinical case study approach (e.g.

36 Moulin et al., 2005; Bancaud et al., 1994) or employed retrospective report to explore individual

37 differences (Martin et al., 2012; O'Connor \& Moulin, 2013). Clinical studies allow the study of

38 déjà vu-like experiences in samples for which they form part of a regularly occurring

39 constellation of symptoms associated with a primary disorder such as dementia or epilepsy.

40 However, the correspondence between clinical and nonclinical manifestations of déjà vu

41 remains unclear, with the potential that they may be mechanistically and subjectively different

42 (e.g. clinical déjà vu associated with dementia, termed déjà vécu, has behavioural

43 consequences - unlike déjà vu in the healthy population, patients with déjà vécu modify their

44 behaviour to avoid the sensation of déjà vu; Moulin et al., 2005). Retrospective reports, whilst

45 affording study of déjà vu in the healthy population, are often recovered weeks or months after

46 the experiences occurred, leaving them open to contamination by bias and reconstruction

47 (Chapman \& Mensh, 1951). Consequently, there has been a recent drive towards developing 


\section{PeerJ Reviewing Manuscript}

48 laboratory-based procedures which reliably generate déjà vu in healthy volunteers. Such

49 laboratory analogues provide the opportunity for the 'here-and-now' study of nonclinical déjà vu experiences and could yield insights into memory decision-making akin to those offered into word-finding by the experimental generation of the tip-of-the-tongue sensation (e.g. Widner,

52 Smith \& Graziano, 1996; Schwartz, 2001).

53 Attempts to find a laboratory analogue of déjà vu have primarily focused on generating 54 sensations of subjective familiarity. For example, Brown and Marsh (2009; building on Jacoby \& 55 Whitehouse, 1989), using subliminal presentation of symbols, and Cleary, Ryals and Nomi 56 (2009), using configural similarity for visual scenes, both generated familiarity in the absence of

57 awareness of its source. The frequency of déjà vu reports stemming from these procedures was 58 high, and the willingness of participants to categorise the experimentally-generated experience 59 as déjà vu likely reflects an overlapping experiential feature, familiarity without recollection.

60 Nevertheless it should be noted that experiences more closely analogous to this experimentally61 generated sensation, at least in their causal mechanism, occur frequently without being labelled 62 as déjà vu e.g. Mandler's (1980) example of the 'butcher on the bus' (formalised in the 63 laboratory as recognition without identification, Cleary, 2006). In the 'butcher on the bus' 64 experience, an individual becomes aware that they recognise someone, but cannot recollect 65 who the person is because the person (the butcher) is being encountered in a different context 66 to that which they were previously encountered (on the bus as opposed to in the supermarket).

67 In the Brown and Marsh (2009) and Cleary et al. (2009) experiments, participants may have 68 misidentified the sensation of recognition without identification as déjà vu because both experiences represent unusual memory sensations where retrieval feels incomplete. Our rationale for continued work towards a laboratory analogue is that a compelling elicitation of déjà

71 vu should attempt to generate all of the components of the experience. In order to do this, we

72 refer again to the definition presented in the first paragraph which incorporates subjective

73 familiarity but also a concurrent awareness of objective unfamiliarity. 
74 The key omission in the déjà vu generation procedures described above is the provision of

75 information allowing the participant to make an evaluation of unfamiliarity or novelty to clash with

76 the experimentally-generated familiarity. In these procedures, there was no objective standard

77 by which participants could verify that the stimuli provoking familiarity had in fact not previously

78 been encountered. With a view to generating a more complete laboratory analogue of

79 naturalistic déjà vu, we developed a procedure during which some stimuli elicit both subjective

80 familiarity and an awareness of novelty. This procedure builds on the DRM recognition task

81 (Deese, 1959; Roediger \& McDermott, 1995) in which participants study a series of words (e.g.

82 rest, bed and blanket) which are all semantically linked to an unpresented word (sleep). This

83 unpresented word, referred to as the critical lure, typically yields illusory recognition when it is

84 presented at test - the critical lure generates a sensation of subjective familiarity. Our procedure

85 pairs the DRM task with an additional task in which participants monitor studied stimuli for a

86 feature present only in the critical lure (e.g. the starting letters 'sle'). When participants become

87 aware of the absence of that feature from the study list words, they also become aware that the

88 critical lure must in fact be novel (see Figure 1). Thus in critical lures, subjective familiarity

89 clashes with an objective awareness of novelty, satisfying the definition of déjà vu.

90 Using this new procedure, we independently varied objective novelty and subjective familiarity,

91 hypothesising that déjà vu reports would be greatest within lists for which the greatest clash

92 between familiarity and novelty was contrived. Assessing déjà vu occurrence on a trial-by-trial

93 basis allowed us to identify the specific word triggers of déjà vu. We hypothesised that déjà vu

94 triggers would be most numerous amongst the words for which maximal familiarity/novelty

95 conflict was generated, critical lures. Finally, we supplemented our categorical déjà vu

96 assessments with discursive responses which we acquired pre- and post-experimentally. We

97 asked participants to write about (i) a previous typical naturalistic experience of déjà vu and (ii)

98 the experimentally-generated experience of déjà vu according to the same criteria. We used 


\section{PeerJ Reviewing Manuscript}

99

100

101

102

103

104

105

106

107

108

109

these responses to better understand the similarities and differences between our experimentally-generated déjà vu experience and naturalistic déjà vu experiences.

\section{Method}

Participants. Thirty English-speaking participants (20 female, 10 male; mean age $=24.1$ years, $S D=6.5$ years) were tested and reimbursed at a rate of $£ 5 /$ hour for their time. Written consent was obtained from all participants. The protocol was approved by the University Teaching and Research Ethics Committee at the University of St Andrews (approval number PS10697).

Stimuli and Materials. Over the course of the experiment, each participant was presented with 24 DRM word lists based on Stadler, Roediger and McDermott's norms (1999). The 24 lists comprised the 12 which yielded the highest false alarm rates for critical lures and the 12 useable lists which yielded the lowest false alarm rates. For each study list, 12 words were randomly selected from the 15 words published per list in Stadler et al. For each test list, 3 old words (targets; selected from the previously studied 12 words) were presented alongside 2 semantically unrelated new words (unrelated lures), 2 semantically related new words (related lures; these were randomly selected from the 3 words excluded from study presentation) and the single critical lure (see Appendix I for four example study-test lists). All word lists were presented on PCs running MATLAB (The MathWorks Inc., Natick, MA, 2000) and Psychophysics Toolbox (Brainard, 1997).

Two paper questionnaires were administered: a pre-experimental questionnaire; and a postexperimental questionnaire. The pre-experimental questionnaire was completed before the first study-test block and assessed participants' previous déjà vu experience (yes/no response) and frequency ( $<1,1-4$ or $>4$, times a year). There followed an explanation of déjà vu based on Brown's (2004) definition, "a feeling of familiarity coupled with the knowledge that this familiarity is incorrect", and an open-ended question requested that participants write a short passage 
124 summing up previous déjà vu experiences according to the following instructions: "Please

125 provide a short account of a 'typical' déjà vu experience you have had. Try to include some

126 detail concerning the following points: Emotional intensity of a typical déjà vu experience;

127 Duration of a typical déjà vu experience; How a déjà vu experience might typically make you

128 feel about the reliability of your memory". The post-experimental questionnaire, completed at the

129 end of the experiment, confirmed participants' experiences of déjà vu during the experiment

130 (yes/no response) and was again followed by an open-ended question requesting a summary of

131 the experimentally-generated experience of déjà vu according to the following instructions:

132 "Please provide a short account of your déjà vu experience(s) during the experiment. Try to

133 include some detail concerning the following points: Emotional intensity of déjà vu

134 experience(s); Duration of déjà vu experience(s); How the déjà vu experience(s) made you feel

135 about the reliability of your memory".

137 Design and Procedure. We manipulated subjective familiarity by presenting participants with

138 DRM lists yielding the highest or the lowest critical lure false alarm rates according to Stadler et 139 al (1999). Lists yielding high false alarm rates were used in the high familiarity conditions. Lists

140 yielding low false alarm rates were used in the low familiarity conditions. We manipulated

141 objective novelty by varying the string that participants monitored study words for. For each

142 study list, participants were presented with a new 1-3 character string, and indicated the number

143 of words which began with this string once they had seen all 12 words. For the high novelty lists,

144 no words in the study list and only the critical lure in the test list began with the character string.

145 For the low novelty lists, at least one word in the study list (and not the critical lure) began with

146 the character string. During each test list, the number of words indicated by participants as

147 beginning with the string was re-presented to participants, highlighting the novelty of critical

148 lures in only the high novelty condition. Thus, there were two within-subjects list-level factors, 149 novelty (high, low) and familiarity (high, low). These combined to produce four types of list: (1)

150 high novelty-high familiarity; (2) high novelty-low familiarity; (3) low novelty-high familiarity; and 
151 (4) low novelty-low familiarity. There was also one within-subjects word-level factor with four

152 levels: targets (previously studied words), unrelated lures (previously unstudied words which

153 were semantically unrelated to the studied words), related lures (previously unstudied words but

154 which were drawn from the same DRM list), and critical lures (previously unstudied words to 155 which all the studied words were semantically related). All four word conditions were presented 156 within each test list.

157 Over the course of the experiment, six study-test list pairs of each list type (high novelty-high 158 familiarity etc.) were presented (24 study-test list pairs in total). To allow participants to rest 159 periodically, the experiment was split into four study-test blocks. The list composition of each 160 block was randomly assigned such that participants would not inevitably encounter each list 161 type in each study-test block. Each study-test block comprised six consecutive study lists, 162 followed by six consecutive test lists. For each participant, corresponding study and test lists 163 were presented in the same order (i.e. study1, study2, study3, study4, study5, study6, test1, 164 test2, test3, test4, test5, test6).

165 Figure 1 shows a schematic of a study list and its corresponding test list. The to-be-monitored 166 character string was presented in size 48pt font alongside word stimuli for the duration of each study list. Twelve words were serially presented in size $48 p t$ font, in the centre of the screen, for 3s each. At the end of the study list, participants were prompted to register the number of words beginning with the character string in that list (0-5 indicated using the keyboard).

170 Throughout each test list, the previously-monitored character string and the number indicated by

171 the participant at the end of the study list were presented in size 48pt font, at the top of the 172 screen. Eight words (three targets, five lures) were then serially presented in size 48pt font, in

173 the centre of the screen. Test words were presented in a pseudorandom order modelled on the 174 procedure used by Roediger \& McDermott (1995). A target always occupied test position 1 and 
175 the critical lure always occupied test position 6,7 or 8 . (The three targets selected comprised

176 the word from study position 1, one word selected at random from study position 2-6 and one

177 from study position 7-12.) The remaining targets and lures were allocated at random to the

178 unoccupied test positions. Below each word, the prompt, "old(b) or new(n)?", presented in size

179 48pt font, cued participants to indicate whether the word was previously presented at study or

180 not. Once a response had been made, a new prompt, "Confidence: $L(1), M(2), H(3)$ ?", cued

181 participants to indicate their confidence in the previous decision using. All test responses were

182 self-paced and responses were made using the keyboard keys listed in parentheses.

183 In addition to the old/new and confidence judgments collected for each test stimulus, we also

184 assessed the occurrence of a déjà vu experience and its intensity using an on-screen toggle

185 system. This response system avoided unnecessarily asking participants about their déjà vu

186 experiences repeatedly, leaving them free to report the experience only when it arose. A déjà vu

187 status bar, located at the bottom of the screen had the default status "deja vu(d): none".

188 Participants reported the occurrence of déjà vu by pressing the 'd' key, which would cycle

189 through the intensity options. On pressing it once, the status would change to "deja vu(d): low".

190 Pressing it again would result in a status change to "deja vu(d): medium" and a third time, to

191 “deja vu(d): high". If the 'd' key was pressed a fourth time, this would restart the cycle at "deja

$192 \mathrm{vu}(\mathrm{d})$ : none". Participants could indicate the occurrence of déjà vu at any point during each trial

193 (i.e. during the self-paced windows for old/new and confidence responding). The status reverted

194 to the default "deja vu(d): none" at the start of each new test word presentation. When

195 participants had completed four study-test blocks they completed the post-experimental

196 questionnaire. The entire procedure lasted no longer than one hour for each participant.

$197 n$-gram Analysis of Déjà vu Descriptions. $n$-grams are continuous sequences of $n$ words

198 found to occur within a passage of prose. $n$-grams with $n=1$ are referred to as unigrams, and

199 those with $n=2$ as bigrams. Examples of unigrams from within this sentence are "sentence" and 
200 "from", whereas examples of bigrams from within this sentence are "of unigrams" and "are

201 sentence". We identified differences in the strings of words used to describe naturalistic and

202 experimentally-generated déjà vu by conducting a rudimentary $n$-gram analysis. The procedures

203 reported here largely mirror those reported in Selmeczy \& Dobbins (2014). Prose passages from

204 the pre-experimental and post-experimental questionnaires underwent identical preparation for

205 n-gram analysis: spelling errors were corrected; contractions were completed (e.g. "don't"

206 became "do not"); symbols with known meanings were written out in full (e.g. "" became "or", "="

207 became "equals"); and "deja vu" replaced with "dejavu".

208 Separately for unigrams and bigrams, we counted the number of times each $n$-gram appeared

209 in the pre- and post-experimental passages. For $n$-grams with total occurrences $(N)$ across both

210 passages of at least the median (unigrams: 5, bigrams: 4), we used $N$, the number of

211 occurrences in the pre-experimental passage, and an assumed binomial-p parameter of .5 to

212 calculate a binomial distribution $z$ value and corresponding $p$ value of each $n$-gram. As we were

213 interested in $n$-grams which differentiated the two accounts, we set an uncorrected $p$ threshold

214 of .05 and tabulated these $n$-grams for examination.

\section{Results}

216 Whilst we do not present a comprehensive analysis of the accuracy data here - we present key

217 analyses in the text and summarise accuracy and confidence for all conditions in Table 1 - we

218 show that the novelty and familiarity component manipulations of the modified DRM task resulted

219 in the expected behavioural changes for recognition accuracy. We then examine the frequency of

220 déjà vu reports, the intensity of déjà vu experiences and finish with a descriptive $n$-gram analysis

221 of the responses to the open-ended questions.

222 Accuracy. To establish that the DRM procedure was indeed generating erroneous familiarity for 223 critical lures, we conducted a one-way repeated measures ANOVA on accuracy according to 
224

225

226

227

228

229

230

231

232

word condition (critical lure, related lure, unrelated lure, target), collapsed across list conditions. Assumptions of sphericity were violated, $X^{2}(5)=11.63, p=.040$, therefore degrees of freedom were corrected with Greenhouse-Geisser estimates of sphericity, using $\varepsilon=.819$. The effect of word condition on accuracy was significant, $F(2.46,71.28)=47.43, p<.001, \eta_{p}^{2}=.621$, with accuracy lowest for critical lures, as anticipated (see Table 1 for descriptives).

To check whether our list manipulations influenced levels of erroneous familiarity, we next conducted a 2 x (novelty: high, low) x 2 (familiarity: high, low) repeated measures ANOVA on critical lure accuracy. There was a significant main effect of novelty, $F(1,29)=24.23, p<.001$, $\eta_{p}^{2}=.455$, such that high novelty critical lures, $M=.714[.617, .811]$, were more accurately responded to than low novelty critical lures, $M=.414[.322, .506]$. There was also a significant main effect of familiarity, $F(1,29)=51.11, p<.001, \eta_{p}{ }^{2}=.638$, such that high familiarity critical lures, $M=.475[.394, .556]$, were less accurately responded to than low familiarity critical lures, $M=.653[.583, .722]$. Finally, there was a significant interaction between novelty and familiarity, $F(1,29)=11.45, p=.002, \eta_{p}^{2}=.283$. Focusing on the main effects, it is evident that both list manipulations had the intended effects on levels of erroneous familiarity generated for critical lures - increased novelty salience decreased erroneous responding by drawing participants' attention to the objective novelty of the critical lure, whilst the lists selected from the Stadler et al (1999) norms for their elevated false alarm rates also demonstrated elevated false alarms in the current procedure.

Déjà vu frequency. Déjà vu was reported at least once by 18 of the 30 participants (60\%). Across all participants, the mean number of déjà vu reports was $12.83[5.85,19.82]$. This value rose to $21.39[11.41,31.37]$ in the subsample who reported at least one déjà vu. In the following analyses, we analyse déjà vu frequency across the whole sample. 
247 Déjà vu occurrence was assessed on a trial by trial basis. We were therefore able to calculate

248 the likelihood with which a word from each condition would yield a déjà vu report (see Table 1

249 and Figure 2A). Déjà vu frequency, as a proportion of all words presented within the given

250 combinations of conditions, was assessed in a 2 (novelty) $\times 2$ (familiarity) $\times 4$ (word) repeated

251 measures ANOVA. Assumptions of sphericity were violated for the main effect of word, $X^{2}(5)=$

$25231.17, p<.001$, and the interactions between word $x$ novelty, $x^{2}(5)=92.60, p<.001$, word $x$

253 familiarity, $X^{2}(5)=54.54, p<.001$, and word $x$ novelty $x$ familiarity, $X^{2}(5)=33.49, p<.001$.

254 Degrees of freedom for these effects were corrected using Greenhouse-Geisser estimates of

255 sphericity, using $\varepsilon=.564, \varepsilon=.413, \varepsilon=.482$ and $\varepsilon=.555$ respectively. Across all effects involving

256 familiarity however, there were no significant differences, all $p s>.350$, suggesting that our

257 manipulation of DRM strength did not influence déjà vu responses independently of the other

258 factors. We therefore present the remaining effects involving the novelty and word conditions

259 below.

260 There was a significant main effect of novelty, $F(1,29)=8.05, p=.008, \eta_{p}^{2}=.217$, with a

261 greater frequency of déjà vu reports under high novelty, $M=.097[.051, .142]$, than low novelty,

$262 M=.064[.027, .101]$. There was also a significant main effect of word, $F(1.69,49.10)=10.28, p$

$263<.001, \eta_{p}^{2}=.262$, driven by the high frequency of déjà vu reports for critical lures (see Table 1

264 for descriptives). ${ }^{1}$ Both of these findings are consistent with our hypotheses. Although there was

265 no graded response according to familiarity condition, the lists contriving salient novelty

266 generated the most déjà vu reports, likely driven by the greatest conflict between DRM-induced

267 familiarity and novelty. Consistent with this interpretation, we were also able to show that déjà vu

268 was reported more for critical lures than any other word condition.

11 The high number of participants reporting no déjà vu experiences ensured that the déjà vu

2 frequency data were highly positively skewed and likely do not satisfy parametric assumptions.

3 We therefore present additional nonparametric tests of the main effects reported above.

4 Wilcoxon's Signed Ranks tests found no effect of familiarity on déjà vu reports, $Z=-1.37, p=$.

5171 , but there was a significant effect of novelty, $Z=-2.74, p=.006$. Friedman's test found a

6 main effect of word, $X^{2}(3)=17.04, p=.001$. Thus, the nonparametric equivalent tests of the

7 main effects matched the patterns of significance obtained from parametric tests. 
269 The novelty $\mathrm{x}$ word interaction was also significant, and likely responsible for both main effects 270 presented above, $F(1.24,35.95)=6.45, p=.011, \eta_{p}{ }^{2}=.182$. Figure 2 illustrates the

271 homogeneity of responding within word conditions, which is broken only for critical lures.

272 Crucially, critical lures in the low novelty condition remained comparable to other word

273 conditions in their likelihood of yielding a déjà vu reports, in the region of $10 \%$, whereas those in

274 the high novelty condition elicited déjà vu responding around $25 \%$ of the time. The presence of

275 salient novelty, whereby participants were made aware that stimuli which they otherwise found

276 to be familiar could not be so, appears important in elevating categorical reports of déjà vu

277 within this procedure.

278 Our hypothesised elevation of déjà vu responses in maximal clash conditions was predicated 279 upon participants correctly identifying critical lures as objectively new. To establish that the 280 described pattern of déjà vu responses persisted in items to which participants had given correct 281 recognition responses but not incorrect recognition responses, we recalculated déjà vu 282 response likelihoods according to recognition response correctness (Figure 2B). Given the 283 ineffectiveness of the familiarity manipulation in influencing déjà vu frequency above, we 284 collapsed across familiarity conditions so as to compare only high and low novelty lists by word 285 condition. In a 2 (novelty) x 4 (word) repeated measures ANOVA on déjà vu responses to 286 correctly identified words, assumptions of sphericity were violated for the main effect of word, $287 X^{2}(5)=41.30, p<.001$, and the interaction, $X^{2}(5)=105.37, p<.001$. Degrees of freedom for 288 these effects were corrected using Greenhouse-Geisser estimates of sphericity, using $\varepsilon=.565$ 289 and $\varepsilon=.385$ respectively. The main effect of novelty was no longer significant, $F(1,26)=3.63, p$ $290=.068, \eta_{p}{ }^{2}=.123$, though the main effect of word survived, $F(1.70,44.11)=8.85, p<.001, \eta_{p}^{2}$

$291=.241$, again carried by the elevated déjà vu responding to critical lures. ${ }^{2}$ The novelty $\mathrm{x}$ word

82 Nonparametric tests of the main effects within correctly identified words found significant

9 effects of both novelty, $Z=-2.74, p=.006$, and word, $X^{2}(3)=12.77, p=.005$. Whilst the 10 nonparametric test significances do not match their parametric equivalents above, they do 
292 interaction was no longer significant, $F(1.16,30.06)=2.16, p=.100, \eta_{p}{ }^{2}=.077$. In an equivalent

293 ANOVA for incorrect responses there were no main effects of novelty, $F(1,12)=3.11, p=.103$,

$294 \eta_{p}{ }^{2}=.206$, or word,,$F<1$, and no significant interaction, $F<1$. $^{3}$ Although the previously

295 described effects are attenuated when split according to recognition response correctness, there

296 is nothing to suggest that the hypothesised elevation in déjà vu responses was driven by

297 responses to critical lures which participants have incorrectly identified as old. There is therefore

298 little indication that the overall findings relating to déjà vu frequency are driven by stimuli to

299 which participants should be reporting no more than baseline levels of déjà vu.

300 Déjà vu intensity. Déjà vu intensity was measured after the presentation of each word and 301 coded as being rated from 1 (low) to 3 (high). We were interested in whether déjà vu, once 302 reported, differed in intensity according to list condition. We again collapsed across familiarity 303 conditions so as to compare déjà vu intensities across high and low novelty lists. We also

304 restricted our analysis to critical lures, the stimuli in which déjà vu reports were most frequent, to

305 avoid the problem of empty cells decimating the analysis. Fourteen participants reported déjà vu

306 under both novelty conditions. A repeated measures t-test found no significant difference

307 between déjà vu intensity ratings for high novelty critical lures, $M=1.67,[1.31,2.03]$, and low

308 novelty critical lures, $M=1.79,[1.38,2.21], t(13)=-0.69, p=.503 d=-.196$. (There were too

309 few participants reporting déjà vu in both novelty conditions to warrant further analysis of the

310 data subdivided according to correct [9 participants] and incorrect [4 participants] recognition

311 responses.) Overall, déjà vu intensity did not vary according to novelty condition.

312 Déjà vu descriptions. In order to quantitatively explore discursive accounts of naturalistic and 313 experimentally-generated déjà vu experiences, we excluded participants who had never

match the parametric and nonparametric significances for the overall data, collapsed across correctness.

133 Nonparametric tests within incorrectly identified words also found nonsignificant effects of

14 novelty, $Z=-0.36, p=.723$, and word, $X^{2}(3)=5.04, p=.169$. 


\section{PeerJ Reviewing Manuscript}

314

315

316

317

318

319

320

\section{1}

322

323

324

325

326

327

328

329

330

331

332

333

334

335

336

337

338

experienced déjà vu or who did not experience déjà vu in the experiment. This left us with a subsample of 15 participants. We conducted $n$-gram analyses on these participants' accounts of naturalistic and experimentally-generated déjà vu. Unigrams and bigrams which significantly differentiate naturalistic and experimental experiences of déjà vu are listed in Table 2, wherein positive $z$ values indicate $n$-grams which were used in the descriptions of previous déjà vu experiences significantly more than the experimental déjà vu experiences. Negative $z$ values indicate $n$-grams which showed the opposite pattern of correspondence.

A common thread across unigrams and bigrams was the presence of words describing the specificity of the déjà vu experience in question. Experimental reports of déjà vu were characterised by descriptions relating to the stimuli ("word[s]", "the word[s]), modality of presentation ("seen", "had seen") and the setting ("experiment", "the experiment") e.g. "Sometimes, I had a very slight feeling that I had seen a particular word before." Naturalistic déjà vu experiences were more generalised (“situation”, "experience”) e.g. "Typically a scenario or situation I am in just seems familiar." In this respect, experimental reports of déjà vu appear to be in response to an experience which is more restricted to certain stimuli within the environment than naturalistic déjà vu experiences.

A related dimension along which there was the suggestion of differentiation was the duration of the experience. "Minutes" was used disproportionately to describe naturalistic experiences e.g. "A typical deja vu experience for me lasts a couple of minutes." "Seconds" appeared in the unthresholded table, but was not diagnostic of one or other category of experience (naturalistic: 4, experimental: $3, z=.352, p=1.00$ ) and was used in similar contexts across accounts e.g. naturalistic - "It lasted only a few seconds..." and experimental - "The deja vu last only a few seconds each time." In general, it would appear that the experimental experience was restricted to a shorter duration than that to which naturalistic déjà vu experiences can extend, though this generalisation did not fit with all participants' experiences e.g. the following from a description of 


\section{PeerJ Reviewing Manuscript}

339 the experimental déjà vu: "The duration was longer than previously experienced deja vu but also

340 fainter."

341 More generally, these accounts offer insights into the nature of the experimentally-generated

342 experience not afforded by the dichotomous or categorical response options available to them in

343 the experiment itself. Specifically, some participants who used the toggle system to indicate that

344 they had experienced déjà vu were much more cautious about describing the experimental

345 experience as déjà vu when given the opportunity to explain their experience more precisely

346 e.g. "I am unsure whether it was exactly deja vu but in some cases I saw words usually words I

347 was expecting to see in the groups but did not, and it felt as if I had seen them." and "I am not

348 really sure I had the two deja vu I reported, or if I think I had them only because it was the task

349 of the experiment." Others reiterated what would be inferred from their categorical responses

350 e.g. "The feeling of deja vu experience is quite strong but I only felt it when certain words came

351 up, when it moved on to the next word... the feeling disappeared..." These accounts raise the

352 question of whether demand characteristics and response acquiescence are important in

353 influencing categorical responses in studies such as this one. Whilst this may not apply to all

354 participants, there are clearly some participants for whom discursive response options give the

355 experimenter a clearer idea of the inferences they should be making based on participant

356 responses.

\section{Discussion}

358 The modified DRM task reliably elicited categorical déjà vu reports. These déjà vu reports varied

359 consistently, largely with our expectations - they were most likely to occur for inappropriately

360 familiar words for which we contrived a clash between illusory familiarity and salient novelty. We

361 showed an increased frequency of déjà vu reports when we elevated the awareness of objective

362 novelty, but not when we elevated strength of DRM-induced familiarity alone. Examination of

363 written accounts allowed us to contrast previously experienced naturalistic experiences of déjà 


\section{PeerJ Reviewing Manuscript}

364 vu with those resulting from the experimental procedures, suggesting that, whilst the

365 experimentally-generated experience may approximate naturalistic déjà vu, it is more restricted

366 in its focus. The written accounts also highlighted the potential influence of demand

367 characteristics in elevating the frequency of déjà vu reports provided in categorical responses

368 alone.

369 Our procedure captures a critical feature of déjà vu, the clash between subjective familiarity and 370 objective novelty (Brown, 2004). Whilst our analyses compared the frequency of déjà vu reports 371 according to the list-wise novelty manipulation, it should also be noted that each list, regardless

372 of the novelty condition, included semantically related lures for which familiarity would have

373 been high and novelty salience was low. Thus, we had both between-list and within-list controls

374 for our high novelty critical lures (critical lures in the low novelty lists and related lures in the high

375 novelty lists respectively). Both of these word conditions were highly familiar yet lacked

376 verifiable novelty and tellingly, yielded significantly lower déjà vu reports than the critical stimuli.

377 Stimuli in either one of these control conditions can be compared to those previously reported

378 as generating déjà vu through familiarity without recollection alone (e.g. Brown \& Marsh, 2009;

379 Cleary et al., 2009) and the baseline levels of déjà vu reported for these stimuli, are consistent

380 with the tendency for participants to report déjà vu under these circumstances. Importantly

381 though, introducing verifiable novelty doubled déjà vu report rates, with this elevation seemingly

382 driven by critical lures correctly identified as novel rather than those about whose objective

383 status participants were confused. It remains to be seen whether contriving objectively verifiable

384 novelty within the alternative procedures would elevate déjà vu responding further, as would be 385 consistent with our operationalisation of the experience.

386 The precise role of objective novelty within the déjà vu experience remains to be established. It 387 may be that novelty is absolutely necessary to establish that the familiarity in question is indeed 388 illusory. Under these circumstances, the inappropriate familiarity signal should be 
389 indistinguishable from a conventional familiarity signal, with déjà vu emerging only from the

390 combination of familiarity and novelty signals indicating that one of them must be wrong.

391 Alternatively, verifiable novelty may help to confirm that the familiarity signal is illusory, though

392 something carried in the familiarity signal alone - some intrinsic indicator that it is inappropriate

393 - may be sufficient to achieve this. That déjà vu in the healthy population has no behavioural

394 consequences and therefore that people tend always to discount the illusory familiarity signal

395 (as opposed to déjà vécu) supports the second alternative. (At this point, it is worth noting that

396 the association between elevated déjà vu responding and increased accuracy to critical lures in

397 the current analogue mirrors the appropriate decision-making that accompanies naturalistic déjà

398 vu experiences in the healthy population.) In any case, verifiable novelty tends to make for a

399 very compelling argument that the familiarity experienced is inappropriate and may therefore

400 lend itself to being told to others and remembered as an archetypal déjà vu experience. Déjà vu

401 for an event which can never have happened before (e.g. Pasteur's funeral, Berrios, 1995) or in

402 a country one has never previously visited (e.g. in France, O'Connor, Lever \& Moulin, 2010) is

403 bound to be more compelling than déjà vu during one's daily commute. Thus, the salient novelty

404 with our experimental analogue may bring it closer to the déjà vu experiences people report to

405 each other, than analogues without this component.

406 Despite the definitional improvement, the current procedure was still unable to generate déjà vu

407 in $40 \%$ of participants, whilst eliciting a large number of déjà vu experiences in the other $60 \%$. It

408 is unclear why the procedure is so variable in its effectiveness. One possibility, which draws on

409 data from the discursive responses, is that some participants may view the experimentally-

410 generated sensation as too restricted in its specificity to warrant being called déjà vu. The

411 restriction to single word stimuli is a key component of the DRM procedure and it is difficult to

412 see how this could be overcome whilst continuing to generate erroneous familiarity in this

413 manner. Nonetheless, the use of secondary tasks to generate verifiable novelty could be

414 successful in other procedures for which richer stimuli have been used to generate reports of 
415 déjà vu or erroneous familiarity (e.g. Cleary et al., 2009; Marsh \& Brown, 2008). A comparison of 416 experimental déjà vu report rates across stimuli of differing richness would offer insight into the 417 degree to which stimulus complexity and coherence affects participants' willingness to report the experience, and therefore the face validity of the experimentally generated sensation itself.

419 The absence of a novelty effect on déjà vu intensity, is also worth considering here. There may 420 be a number of reasons for this finding including: i) that questions about déjà vu intensity are 421 responded to more conservatively than questions about déjà vu occurrence; ii) that once 422 elicited, déjà vu intensity does not correspond to the strength of its trigger to elicit frequent déjà 423 vu reports; and relatedly iii) that déjà vu is an 'all-or-nothing' categorical process. That there 424 were no floor effects in the compared intensity ratings argues against explanation $\mathrm{i}$. The other 425 explanations however, warrant further investigation. Explanation ii could be falsified relatively 426 easily. With refinement of the current procedure to generate déjà vu at varying frequencies, 427 intensity ratings could be collected at each level, with a view to establishing a relationship 428 between the two variables. The presence of a frequency-intensity relationship at some levels of 429 frequency would suggest that the null finding here is caused by a failure to calibrate the current 430 search appropriately. The absence of a relationship across all levels would suggest that the 431 relationship between déjà vu intensity and the likelihood of déjà vu generation is not 432 straightforward - a correspondence between the two continuous variables could either be 433 absent or, according to explanation iii, impossible. In this and previous work conducted in the 434 lab, participants have been able to quantify the intensity of their déjà vu experiences on a 435 continuous scale indicating that, counter to explanation iii, déjà vu is not subjectively 436 experienced as categorical. Similarly though, other memory experiences which are often 437 conceptualised as categorical can be continuously quantified by participants (e.g. recollection; 438 Yonelinas, 1994; Mickes, Wais, Wixted, 2009). Thus the nature of the déjà vu experience, as 439 categorical or continuous in its presence and intensity, remains to be fully established. 
440 Within this report, we have largely referred to the analysis of discursive responses as a

441 counterpoint to the categorical self-reports of déjà vu collected during the modified DRM task.

442 However, our $n$-gram analyses are also prone to bias from the question used to generate

443 discursive accounts, which we may have introduced by asking participants about a "typical" past

444 experience of déjà vu. Instead of detailing a specific episode which typifies their experience of

445 déjà vu, many participants spoke in general terms about typical déjà vu experiences, which will

446 undoubtedly have influenced our comparison of the two accounts. We acknowledge that this

447 method could be improved by simply modifying the question asked, but also suggest that further

448 developments to this procedure may be valuable, especially in an attempt to better understand

449 the subjective experience beyond simple extrapolation from categorical responses to questions

450 about such unusual experiences. One such approach is to train support vector machines

451 (SVMs) to more objectively classify discursive responses as belonging to one or other category

452 of experience. This approach requires a larger corpus of text than was collected in this

453 experiment, but which could be obtained if participants were asked to describe a greater number

454 of previous and experimentally generated déjà vu experiences. Selmeczy and Dobbins (2013)

455 successfully applied SVMs to demonstrate that linguistic content differs according to the

456 recognition memory processes engaged at retrieval and we suggest that such approaches could

457 also be applied to the study of déjà vu experiences.

458 Finally, despite progress towards a viable laboratory analogue, the pattern of déjà vu reports 459 from the current experiments highlights a pervasive, problematic issue within the field. Whilst 460 déjà vu was significantly more likely to be reported for critical lures, it was nonetheless also 461 reported for other words. O'Connor and Moulin (2010) suggest that such non-hypothesised 462 reporting (and therefore a proportion of hypothesised reporting) is driven by demand 463 characteristics (Orne, 1962), a point highlighted by one participant in their discursive response.

464 To minimise this artifact, O'Connor and Moulin suggested that déjà vu be assessed by post465 experimental questionnaire alone, thereby reducing the trial-by-trial suggestion that déjà vu 
466 should be occurring. We found this impractical when seeking to identify specific words triggers 467 of déjà vu reports, and implemented a toggle system using which participants could ignore the 468 question of déjà vu occurrence until it became pertinent. Nonetheless, the persistent cue may 469 still have acted to reinforce acquiescent responding. Alternative methods of questioning which

470 afford both trial-level specificity and minimal pressure to acquiesce would add further credibility

471 to reports proposing laboratory analogues of déjà vu. In their absence however, reporting

472 procedures which allow participants to contextualise their responses go some way towards

473 clarifying the features of a naturalistic experience that are both well and poorly represented by

474 analogues such as this.

\section{Author Contributions}

476 Both authors developed and designed the experiment. Urquhart tested participants. Urquhart 477 analysed and interpreted data under O'Connor's supervision. Urquhart drafted the manuscript 478 and O'Connor provided revisions. Both authors approved the final version of the manuscript for 479 submission.

\section{Acknowledgements}

481 We are thankful to Ronan Kearney for his work prompting development of the déjà vu toggle 482 procedure. 
483

484

485

486

487

488

489

490

491

492

493

494

495

496

497

498

499

500

501

502

503

504

505

506

507

508

\section{References}

Bancaud, J., Brunet-Bourgin, F, Chauvel, P, \& Halgren, E. (1994). Anatomical origin of déjà vu and vivid 'memories' in human temporal lobe epilepsy. Brain, 117(1), 71-90. doi: 10.1093/brain/117.1.71

Berrios, G.E. (1995) Déjà vu in France during the 19th century: A conceptual history. Comprehensive Psychiatry, 26(2), 123-129. doi: 10.1016/S0010-440X(95)90107-8

Brainard, D.H. (1997). The Psychophysics Toolbox. Spatial Vision, 10, 433-436. doi: $10.1163 / 156856897 \times 00357$

Brown, A.S. (2003). A review of the déjà vu experience. Psychological Bulletin, 129(3), 394-413. doi: $10.1037 / 0033-2909.129 .3 .394$

Brown, A.S. (2004). The déjà vu experience. New York: Psychology Press.

Brown, A.S. \& Marsh, E.J. (2008). Evoking false beliefs about autobiographical experience. Psychonomic Bulletin \& Review, 15(1), 186-190. doi: 10.3758/pbr.15.1.186

Brown, A.S., \& Marsh, E.J. (2009). Creating illusions of past encounter through brief exposure. Psychological Science, 20, 534-538. doi: 10.1111/j.1467-9280.2009.02337.x

Chapman, A.H., \& Mensh, I.N. (1951). Déjà vu experience and conscious fantasy in adults. Psychiatric Quarterly Supplement, 25, 163-175.

Cleary, A.M. (2006). Relating fam-based recognition and the tip-of-the-tongue phenomenon: Detecting a word's recency in the absence of access to the word. Memory \& Cognition, 34, 804-816. doi: 10.3758/BF03193428

Cleary, A.M., Ryals, A.J., \& Nomi, J.S. (2009). Can déjà vu result from similarity to a prior experience? Support for the similarity hypothesis of déjà vu. Psychonomic Bulletin \& Review, 16, 1082-1088. doi: 10.3758/PBR.16.6.1082

Deese, J. (1959). On the prediction of occurrence of particular verbal intrusions in immediate recall. Journal of Experimental Psychology, 58, 17-22. doi: 10.1037/h0046671

Heller, J. (1961). Catch 22. New York: Simon \& Schuster. 
509 Jacoby, L.L., \& Whitehouse, K. (1989). An illusion of memory: False recognition influenced by

510 unconscious perception. Journal of Experimental Psychology: General, 118, 126-135. doi:

$511 \quad 10.1037 / 0096-3445.118 .2 .126$

512 Martin, C.B., Mirsattari, S.M., Pruessner, J.C., Pietrantonio, S., Burneo, J.G., Hayman-Abello, B.

513 \& Köhler S. (2012). Déjà vu in unilateral temporal-lobe epilepsy is associated with

514 selective familiarity impairments on experimental tasks of recognition memory.

$515 \quad$ Neuropsychologia, 50(13), 2981-2991. doi: 10.1016/j.neuropsychologia.2012.07.030

516 Mandler, G. (1980). Recognizing: The judgment of previous occurrence. Psychological Review,

$517 \quad 87,252-271$. doi: 10.1037/0033-295X.87.3.252

518 Mickes, L., Wais, P.E., \& Wixted, J.T. (2009). Recollection Is a Continuous Process: Implications

519 for Dual-Process Theories of Recognition Memory. Psychological Science, 20(4), 509-515.

520 doi: $10.1111 / \mathrm{j} .1467-9280.2009 .02324 . x$

521 Moulin, C.J.A., Conway, M.A., Thompson, R.G., James, N., \& Jones, R.W. (2005). Disordered

522 memory awareness: recollective confabulation in two cases of persistent déjà vecu.

523 Neuropsychologia, 43(9), 1362-1378. doi: 10.1016/j.neuropsychologia.2004.12.008

524 O'Connor, A.R., Lever, C. \& Moulin, C.J.A. (2010). Novel insights into false recollection: A model

525 of déjà vécu. Cognitive Neuropsychiatry, 15, 118-144. doi: 10.1080/13546800903113071

526 O'Connor, A.R. \& Moulin, C.J.A (2010). Recognition Without Identification, Erroneous Familiarity,

527 and Déjà Vu. Current Psychiatry Reports, 12, 165-173. doi: 10.1007/s11920-010-0119-5

528 O'Connor, A.R. \& Moulin, C.J.A. (2013). Déjà vu experiences in healthy subjects are unrelated

529 to laboratory tests of recollection and familiarity for word stimuli. Frontiers in Psychology

$530 \quad$ 4:881. doi: $10.3389 /$ fpsyg. 2013.00881

531 Orne, M.T. (1962). On the social psychology of the psychological experiment: with particular

532 reference to demand characteristics and their implications. American Psychologist, 17,

533 776-783. doi: 10.1037/h0043424 
534 Roediger, H.L., \& McDermott, K. B. (1995). Creating false memories: Remembering words not

535 presented in lists. Journal of Experimental Psychology: Learning, Memory and Cognition,

536 24(4), 803-814. doi: 10.1037/0278-7393.21.4.803

537 Schwartz, B.L. (2001). The relation of tip-of-the-tongue states and retrieval time. Memory \&

538 Cognition, 29, 117-126. doi: 10.3758/BF03195746

539 Selmeczy, D. \& Dobbins, I.G. (2014). Relating the Content and Confidence of Recognition

540 Judgments. Journal of Experimental Psychology: Learning, Memory, and Cognition, 40(1),

$541 \quad 66-85$. doi: $10.1037 / \mathrm{a} 0034059$

542 Silver, J. (Producer), Wachowski, A. (Director), Wachowski, L. (Director). (1999). The Matrix

543 [Motion picture]. USA: Warner Bros.

544 Stadler, M.A., Rodiger, H.L. \& McDermott, K.B. (1999). Norms for word lists that create false

545 memories. Memory \& Cognition, 27(3), 494-500. doi: 10.3758/BF03211543

546 Widner, R.L., Smith, S.M., \& Graziano, W.G. (1996). The effects of demand characteristics on

547 the reporting of tip-of-the-tongue and feeling-of-knowing states. American Journal of

$548 \quad$ Psychology, 109, 525-538. doi: 10.2307/1423392

549 Yonelinas, A.P. (1994). Receiver-operating characteristics in recognition memory: Evidence for a 550 dual-process model. Journal of Experimental Psychology: Learning, Memory, and

551 Cognition, 20, 1341-1354. doi: 10.1037/0278-7393.20.6.1341 


\section{Table $\mathbf{1}_{\text {(on next page) }}$}

Accuracy and confidence for old/new judgements and déjà vu likelihood according to condition

Upper-case $\mathrm{N}$ indicates high novelty lists, lower-case $\mathrm{n}$ indicates low novelty lists. Upper-case $F$ indicates high familiarity lists, lower-case $f$ indicates low familiarity lists. Accuracy is expressed as the proportion of correct responses. Confidence is expressed as the mean confidence in recognition response accuracy, where response options 'low', 'medium' and 'high' were coded 1, 2 and 3 respectively. Déjà vu indicates déjà vu likelihood, expressed as the proportion of words eliciting a report of déjà vu (of any intensity). In all cells, means are shown, followed by $95 \%$ Cls in brackets. 


\begin{tabular}{lccccc}
\hline \multicolumn{7}{c}{$\mathrm{N} / \mathrm{F}$} & $\mathrm{N} / \mathrm{f}$ & $\mathrm{n} / \mathrm{F}$ & $\mathrm{n} / \mathrm{f}$ & Overall \\
\hline Accuracy & & & & \\
$\quad$ Critical Lure & $.667[.561, .773]$ & $.761[.662, .860]$ & $.283[.182, .384]$ & $.544[.446, .642]$ & $.564[.493, .635]$ \\
$\quad$ Related Lure & $.731[.653, .808]$ & $.811[.739, .883]$ & $.769[.703, .836]$ & $.839[.776, .902]$ & $.788[.731, .844]$ \\
$\quad$ Unrelated Lure & $.925[.882, .969]$ & $.933[.897, .969]$ & $.947[.914, .980]$ & $.928[.888, .968]$ & $.933[.903, .964]$ \\
$\quad$ Target & $.759[.707, .812]$ & $.759[.707, .812]$ & $.754[.694, .813]$ & $.774[.715, .833]$ & $.762[.715, .808]$ \\
$\quad$ Overall & $.770[.727, .814]$ & $.816[.772, .861]$ & $.688[.649, .728]$ & $.771[.732, .810]$ & $.762[.725, .798]$ \\
Confidence & & & & \\
$\quad$ Critical Lure & $2.34[2.14,2.54]$ & $2.50[2.30,2.70]$ & $1.93[1.75,2.12]$ & $1.97[1.78,2.17]$ & $2.19[2.02,2.35]$ \\
$\quad$ Related Lure & $1.98[1.79,2.17]$ & $2.01[1.82,2.21]$ & $1.97[1.79,2.14]$ & $2.01[1.83,2.19]$ & $1.99[1.82,2.17]$ \\
$\quad$ Unrelated Lure & $2.36[2.16,2.56]$ & $2.34[2.14,2.53]$ & $2.43[2.24,2.61]$ & $2.33[2.16,2.51]$ & $2.36[2.18,2.54]$ \\
$\quad$ Target & $2.25[2.07,2.42]$ & $2.39[2.23,2.54]$ & $2.30[2.14,2.46]$ & $2.42[2.26,2.57]$ & $2.34[2.19,2.49]$ \\
$\quad$ Overall & $2.23[2.06,2.41]$ & $2.31[2.15,2.47]$ & $2.16[2.00,2.31]$ & $2.18[2.03,2.34]$ & $2.22[2.07,2.37]$ \\
Déjà vu & & & & \\
$\quad$ Critical Lure & $.222[.095, .349]$ & $.250[.127, .373]$ & $.111[.032, .190]$ & $.111[.041, .181]$ & $.174[.088, .259]$ \\
$\quad$ Related Lure & $.075[.027, .123]$ & $.064[.016, .111]$ & $.061[.021, .101]$ & $.069[.029, .110]$ & $.067[.028, .107]$ \\
$\quad$ Unrelated Lure & $.011[.000, .022]$ & $.025[.005, .045]$ & $.025[.005, .045]$ & $.006[-.002, .013]$ & $.017[.004, .029]$ \\
$\quad$ Target & $.076[.019, .133]$ & $.052[.000, .104]$ & $.072[.021, .124]$ & $.057[.004, .111]$ & $.064[.013, .116]$ \\
$\quad$ Overall & $.096[.049, .143]$ & $.098[.052, .143]$ & $.067[.027, .108]$ & $.061[.026, .096]$ & $.080[.041, .120]$ \\
\hline 1 & & & & &
\end{tabular}




\section{Table 2 (on next page)}

n-grams differentiating naturalistic and experimentally-generated déjà vu

The $n$-gram column shows unigrams and bigrams which a) occurred with a frequency of at least the median ( 5 for unigrams, 4 for bigrams) across all text within the descriptions of naturalistic and experimental déjà vu occurrences and b) were significantly

disproportionately represented $(p<.05)$ in one or other set of descriptions. The naturalistic and experimental headings quantify occurrences of the $\mathrm{n}$-grams within the corresponding set of descriptions across all amalgamated accounts. $N$ is the total count across both sets of descriptions, $z$ is the binomial distribution $z$ value calculated using the listed $n$-gram frequencies and an assumed binomial-p parameter of .5 , and $p$ is the probability of obtaining this $z$ value by chance. 


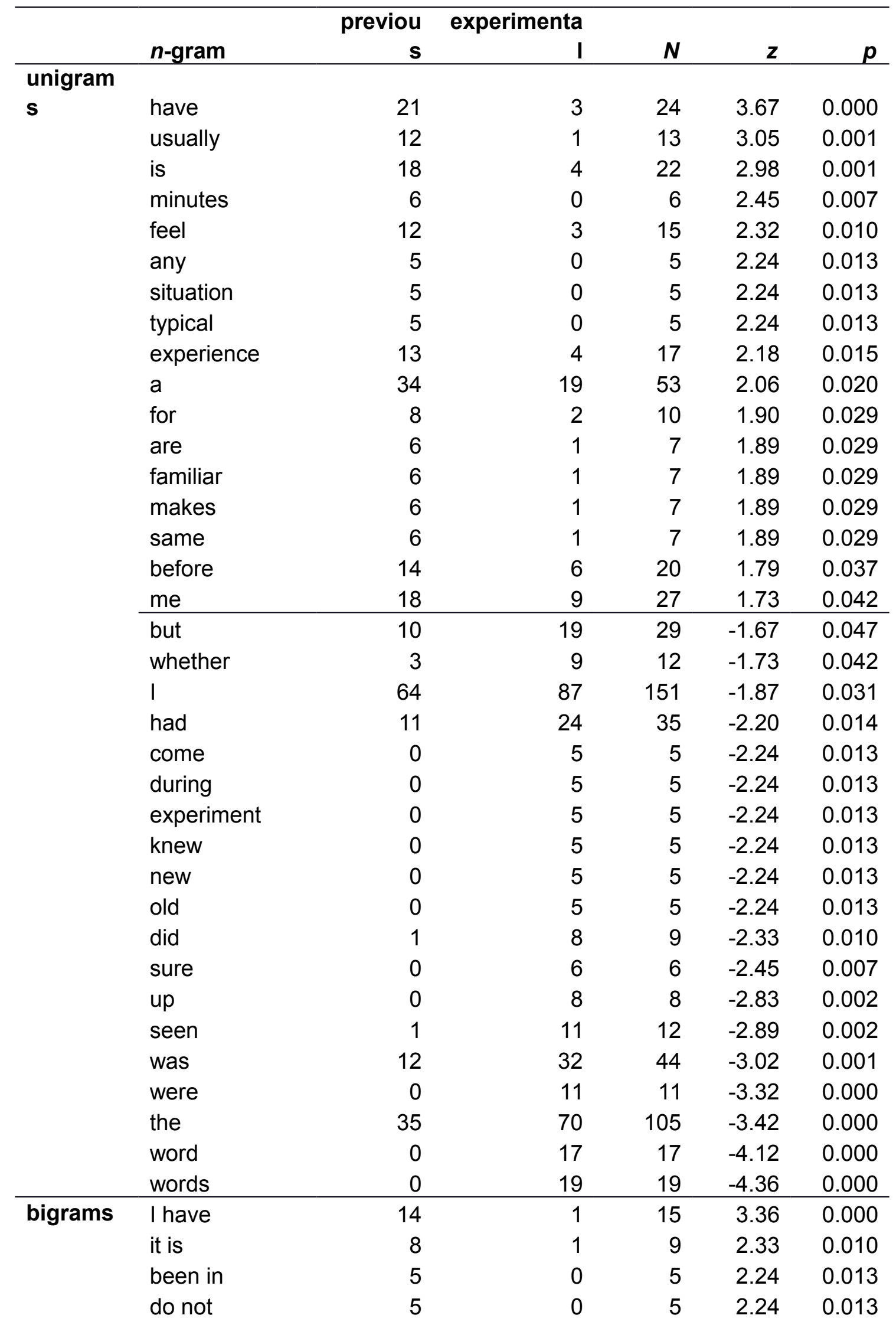




\begin{tabular}{llrrrr} 
is not & 5 & 0 & 5 & 2.24 & 0.013 \\
a typical & 4 & 0 & 4 & 2.00 & 0.023 \\
it usually & 4 & 0 & 4 & 2.00 & 0.023 \\
that my & 4 & 0 & 4 & 2.00 & 0.023 \\
think that & 4 & 0 & 4 & 2.00 & 0.023 \\
have been & 6 & 1 & 7 & 1.89 & 0.029 \\
makes me & 6 & 1 & 7 & 1.89 & 0.029 \\
\hline come up & 0 & 4 & 4 & -2.00 & 0.023 \\
I would & 0 & 4 & 4 & -2.00 & 0.023 \\
the experiment & 0 & 4 & 4 & -2.00 & 0.023 \\
the feeling & 0 & 4 & 4 & -2.00 & 0.023 \\
the list & 0 & 4 & 4 & -2.00 & 0.023 \\
what i & 0 & 4 & 4 & -2.00 & 0.023 \\
word was & 0 & 4 & 4 & -2.00 & 0.023 \\
words i & 0 & 4 & 4 & -2.00 & 0.023 \\
words that & 0 & 4 & 4 & -2.00 & 0.023 \\
during the & 0 & 5 & 5 & -2.24 & 0.013 \\
I knew & 0 & 5 & 5 & -2.24 & 0.013 \\
did not & 0 & 6 & 6 & -2.45 & 0.007 \\
had not & 0 & 6 & 6 & -2.45 & 0.007 \\
had seen & 0 & 6 & 6 & -2.45 & 0.007 \\
it was & 1 & 9 & 10 & -2.53 & 0.006 \\
the words & 0 & 7 & 7 & -2.65 & 0.004 \\
in the & 1 & 10 & 11 & -2.71 & 0.003 \\
I had & 5 & 20 & 25 & -3.00 & 0.001 \\
the word & 0 & 13 & 13 & -3.61 & 0.000 \\
\hline
\end{tabular}




\section{Figure 1}

\section{Schematic of study and test lists}

A possible study and test list for a high familiarity DRM list with critical lure 'sleep'. At the start of the study list, participants were presented with a question reminding them to monitor the study list for words beginning with a character string. The character string remained onscreen throughout the study list. In this case, the high novelty condition string was 'SLE' and the low novelty condition string was ' $\mathrm{B}$ '. Participants were then presented with 12 words semantically related to the unpresented critical lure, each word presented at $3 \mathrm{~s}$ intervals. At the end of the study list, participants indicated the number of words presented which began with the character string. In high novelty conditions, the correct answer was always ' 0 ', in low novelty conditions the correct answer always greater than 0 . Six study lists were presented in each study phase before the test phase was initiated. At the start of each test list, participants were reminded of the previously monitored string and the number of words they reported at study beginning with this string. The reminder remained onscreen throughout the eight-word test list. For each test trial, participants first indicated whether the word had been presented at study (old) or not (new) and then indicated their confidence in this decision. Throughout both of these self-paced decisions, participants could toggle their déjà vu response from 'none' through 'low', 'medium' and 'high'. Once participants made a confidence decision the next test trial was initiated. In the schematic, different word conditions are shown bounded by different coloured boxes and the reminder information is omitted from test words 2-8 for the sake of clarity. In the experiment there was no differentiation of stimulus type visible to participants. The six test lists corresponding to the six study lists from the preceding study phase were presented in the test phase. Over the entire experiment, there were four study-test blocks. 


\section{Study}
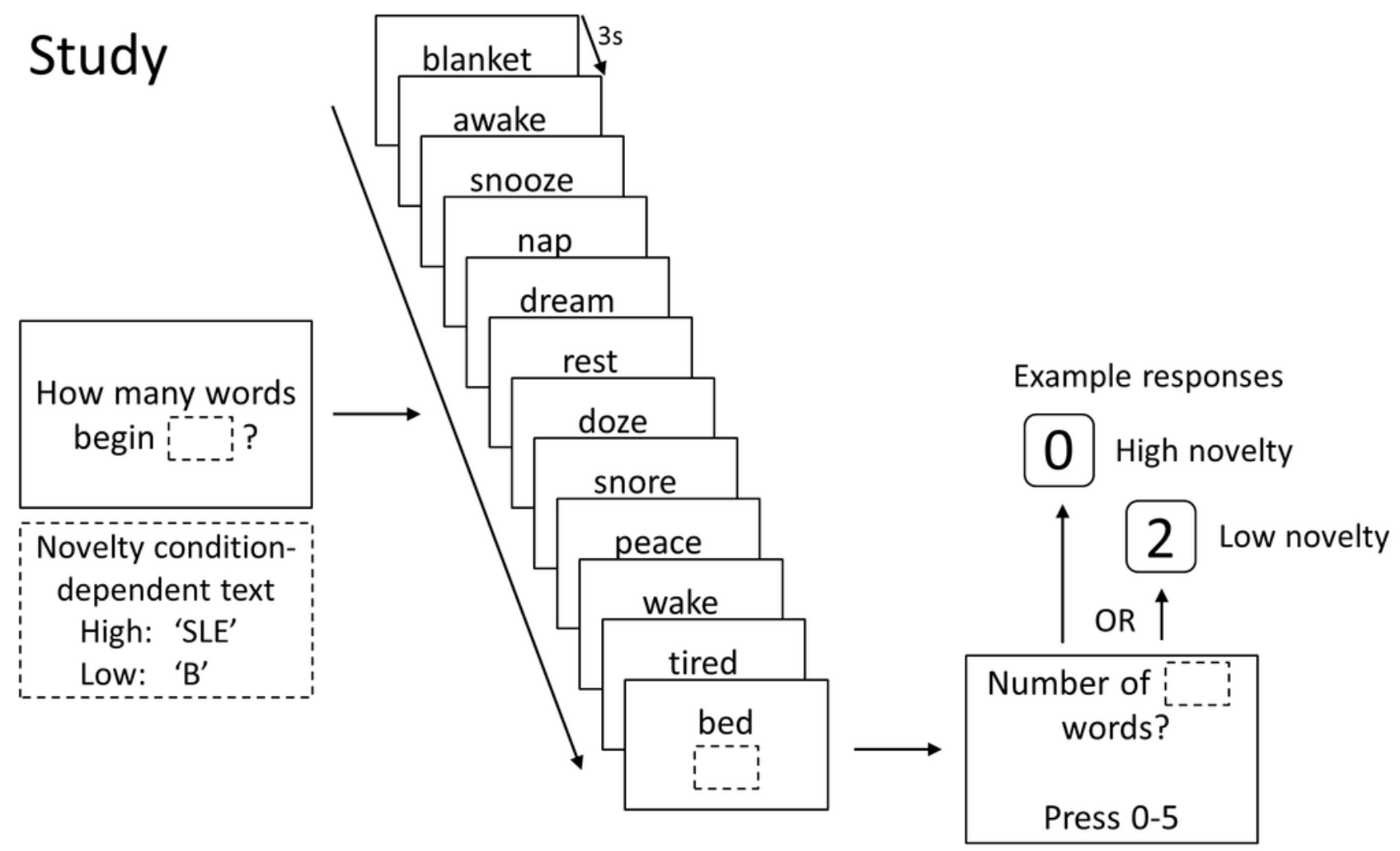

Example responses

Test
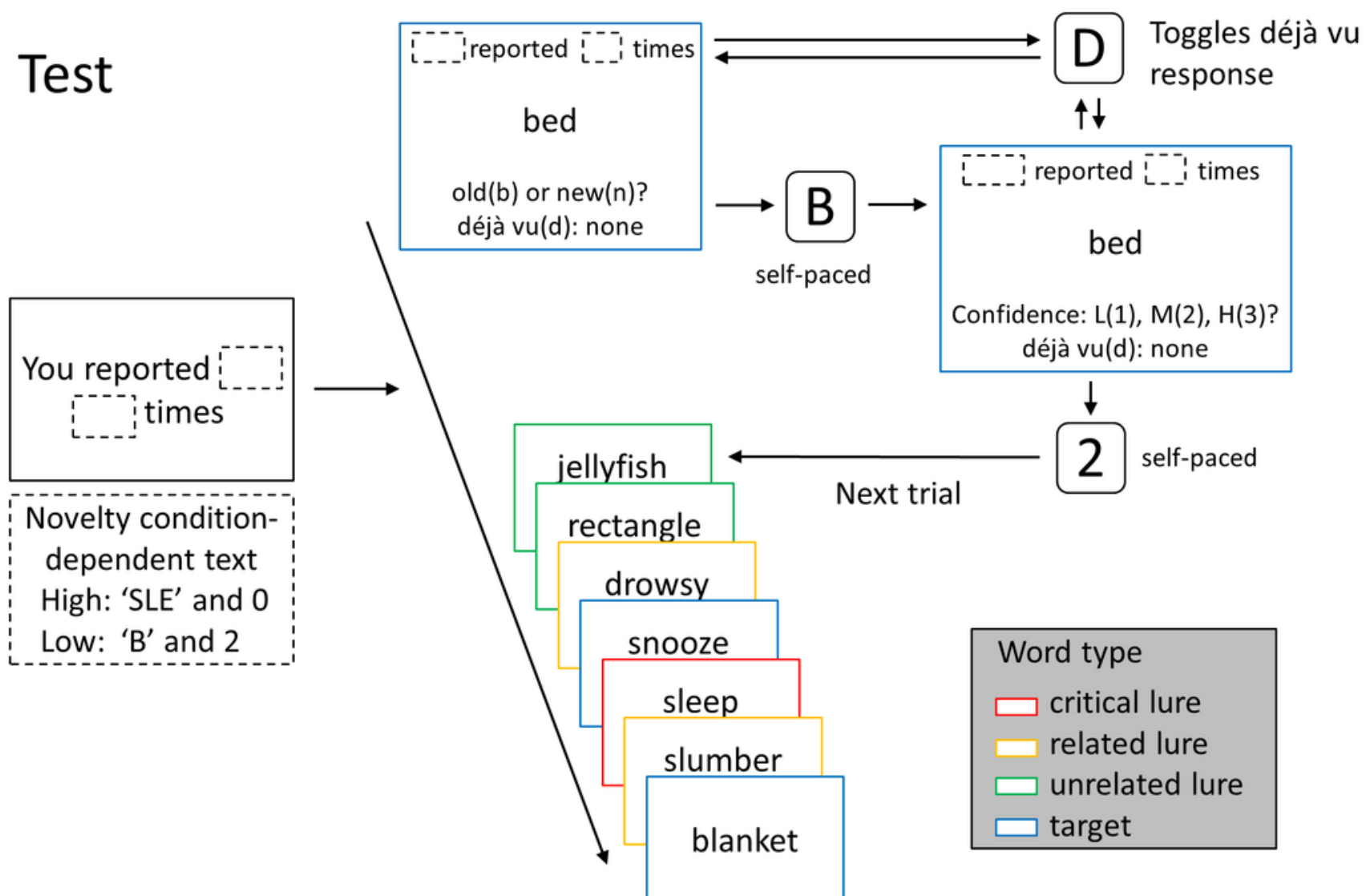


\section{Figure 2}

Likelihood of déjà vu responding according to list and word condition

Panel A shows the likelihood of a déjà vu response according to list and word type collapsed across recognition response correctness. Upper-case $\mathrm{N}$ indicates high novelty lists (blue hues), lower-case $n$ indicates low novelty lists (orange hues). Upper-case $F$ indicates high familiarity lists, lower-case $f$ indicates low familiarity lists. Panel B shows the likelihood of a déjà vu response according to novelty manipulation ( $\mathrm{N}$ is high novelty lists [blue], $\mathrm{n}$ is low novelty lists [orange]) and word type ( $\mathrm{cl}$ is critical lure, $\mathrm{rl}$ is related lure, $\mathrm{ul}$ is unrelated lure, $\mathrm{t}$ is target), split according to correct (left panel) and incorrect (right panel) recognition responding. Error bars represent $95 \% \mathrm{Cls}$. 
A

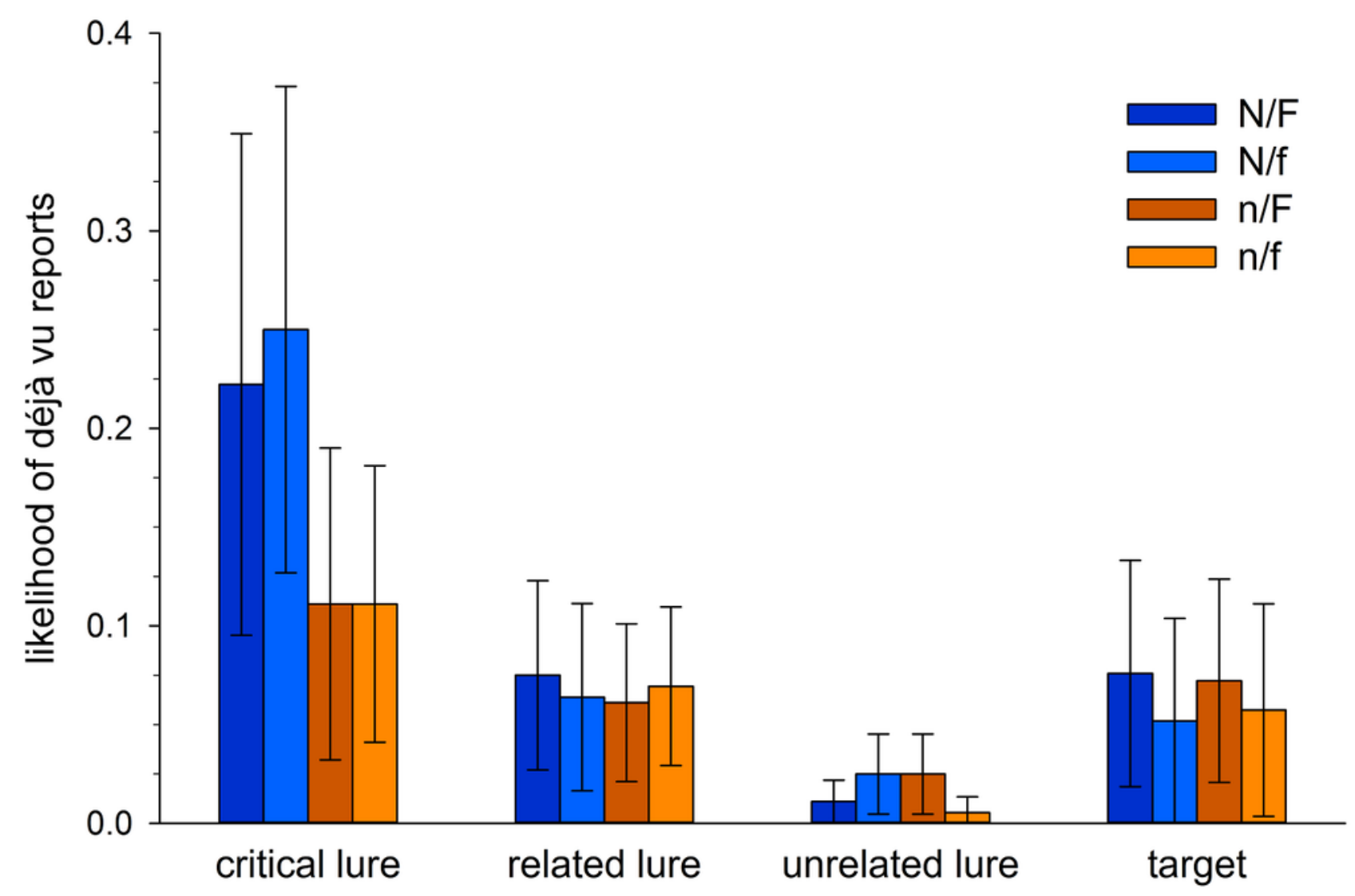

B

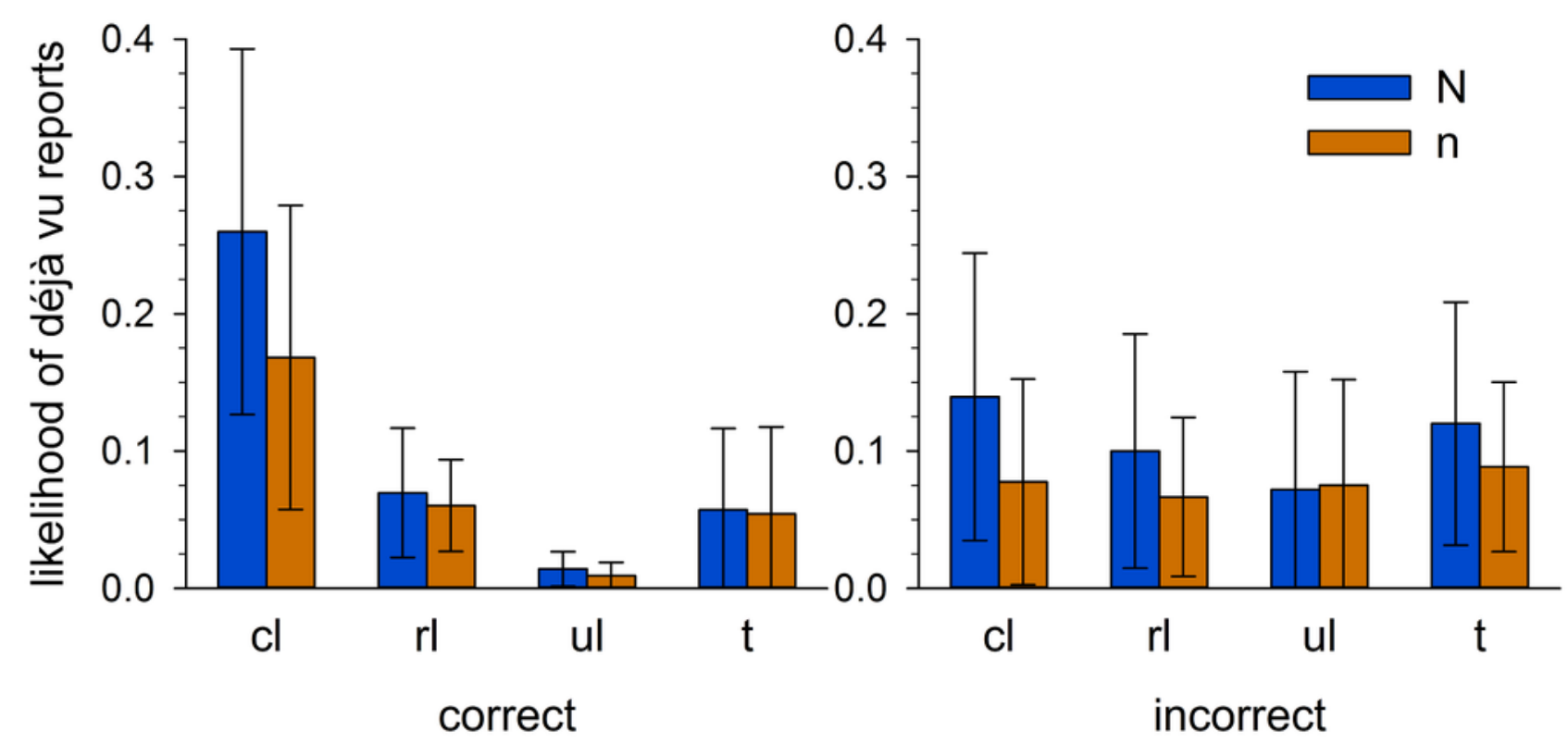

